\title{
Nanocrystallization and amorphization induced by reactive nitrogen sputtering in iron and permalloy
}

\author{
Rachana Gupta and Mukul Gupta* \\ Laboratory for Neutron Scattering, ETHZ \& PSI, \\ Paul Scherrer Institut, Villigen, CH-5232, Switzerland
}

(Dated: August 15, 2018)

\begin{abstract}
Thin films of iron and permalloy $\left(\mathrm{Ni}_{80} \mathrm{Fe}_{20}\right)$ were prepared using an $\mathrm{Ar}+\mathrm{N}_{2}$ mixture with magnetron sputtering technique at ambient temperature. The nitrogen partial pressure, during sputtering process was varied in the range of $0 \leq \mathrm{R}_{N 2} \leq 100 \%$, keeping the total gas flow at constant. At lower nitrogen pressures $\left(\mathrm{R}_{N_{2}} \leq 33 \%\right)$ both $\mathrm{Fe}$ and $\mathrm{NiFe}$, first form a nanocrystalline structure and an increase in $\mathrm{R}_{N 2}$, results in formation of an amorphous structure. At intermediate nitrogen partial pressures, nitrides of Fe and NiFe were obtained while at even higher nitrogen partial pressures, nitrides themselves became nanocrystalline or amorphous. The surface, structural and magnetic properties of the deposited films were studied using x-ray reflection and diffraction, transmission electron microscopy, polarized neutron reflectivity and using a DC extraction magnetometer. The growth behavior for amorphous film was found different as compared with poly or nanocrystalline films. The soft-magnetic properties of FeN were improved on nanocrystallization while those of $\mathrm{NiFeN}$ were degraded. A mechanism inducing nanocrystallization and amorphization in $\mathrm{Fe}$ and $\mathrm{NiFe}$ due to reactive nitrogen sputtering is discussed in the present article.

PACS numbers: 81.15.Cd, 68.55.Jk, 68.60.Dv, 61.10.Kw, 61.46.+w, 75.70.-i
\end{abstract}

\section{INTRODUCTION}

During recent years, nanostructured and amorphous thin films and multilayers of magnetic materials have attracted tremendous scientific and technological interests due to their unique properties compared to conventional crystalline materials 1.2 .3 .4 In nanocrystalline materials, as the grain size decreases, there is a significant increase in the volume fraction of grain boundaries or interfaces. This characteristic strongly influences the chemical and physical properties of the material. In particular, a decrease in the grain size results in improved soft-magnetic properties. On the other hand, amorphous phases are expected to be free from grains and grain-boundaries which often results in release of intrinsic stresses, decrease in magnetic anisotropy and a smoother surface or interface. Furthermore, grains or grain-boundaries act like active path for diffusion, and therefore, atomic self-diffusion in amorphous phases is expected to be lower. Amorphous or nano grain thin films exhibit a short range ordering in the microstructure and their structural, mechanical, electrical, and magnetic properties often depends on the methods and conditions of preparation. $5.6,7$ Various attempts have been made to achieve amorphization in binary or multicomponent metal-metal and metal-metalloid systems using different techniques such as rapid-melt quenching, 8 mechanical alloying, $\frac{9}{9}$ hydrogenation,,$\frac{10}{1}$ pressure,, 11 interdiffusion reaction ${ }^{12,13,14}$ and ion or electron irradiation. ${ }^{15,16}$

Quite recently, nitrogen reactive sputtering has also been used to achieve a nanocrystalline or amorphous phase $17,18,19,20$ In the sputtering process the adatoms have energy of the order of few tens of $\mathrm{eV}$,and during condensation onto the substrate,adatoms are quenched and may form an amorphous or fine grain structure. At the same time, when sputtered using low $\mathrm{Z}$ reactive ions e.g. nitrogen ions, they may occupy interstitial sites in the unit cell of sputtered species, causing a distortion of the unit cell. A combined effect of these situations may lead to a nanocrystalline or amorphous structure of the deposited film. In order to verify such a mechanism causing nanocrystallization or amorphization, two different materials namely bcc Fe and fcc NiFe permalloy were chosen for the present study. In earlier studies, Fe thin films have been prepared using an $\mathrm{Ar}+\mathrm{N}_{2}$ gas mixture by magnetron sputtering $\stackrel{21.22}{2}$ rf sputtering $\stackrel{23.24}{~ p u l s e d ~}$ laser deposition, 25.26.27 ion-beam enhanced deposition (IBED), 28 etc. The motivation of most of these studies was to obtain nitrogen poor $\mathrm{Fe}_{16} \mathrm{~N}_{2}$ phase which possess very high magnetic moment. ${ }^{29.30}$ In some of these studies an amorphous or nanocrystalline phase of $\mathrm{FeN}$ was obtained at low nitrogen pressure ${ }^{23.24 .28}$ However, a detailed investigation of evolution of nanocrystalline or amorphous phases and a mechanism inducing nanocrystallization or amorphization was not studied. It is known that when heated, evaporated, ablated or sputtered in nitrogen environment or with nitrogen ion, iron forms a microstructure with a variety of FeN alloys and compounds, including the recently discovered newcubic-type nitrides.21.26 Ferromagnetic nitrides of iron have received tremendous interests in magnetic functional devices ${ }^{31.32} \mathrm{On}$ the other hand, NiFe alloy with a composition of $\mathrm{Ni}_{80} \mathrm{Fe}_{20}$ is a well-known soft-magnetic alloy and is known as permalloy. It forms a face centered cubic structure of the type $\mathrm{Ni}_{3} \mathrm{Fe}$. In a recent study by Chiba et al., 33 NiFe nitrides were deposited using rf sputtering technique for a nitrogen flow in the range of $5-30 \%$. A decrease in saturation magnetization is reported, however a detailed variation in microstructure with higher nitrogen content was not investigated. 
In present work our aim is to explore the structural and magnetic properties and growth behavior of the bcc $\mathrm{Fe}$ and $\mathrm{fcc} \mathrm{NiFe}$ thin films prepared using reactive nitrogen sputtering in the whole nitrogen partial pressure range $(0-100 \%)$. In a study by Kawamura et al ${ }^{34}$ thin films of NiN were studied. In the present case it was found that both $\mathrm{Fe}$ and $\mathrm{NiFe}$, forms an amorphous or nanocrystalline phase of either the element or a nitride of them when sputtered with a nitrogen poor or rich mixtures. Polycrystalline films containing a mixture of nitrides were obtained at intermediate gas pressures and below or above, the long range ordering of either the pure metal or nitrides of them is restricted and a nanocrystalline or amorphous structure is obtained. On the basis of obtained results a mechanism leading the breakdown of long range ordering is discussed. In order to understand the physical properties of the formed amorphous phases, crystallization process was studied after annealing the thin films in vacuum. It is known that amorphous films have a smoother surface, due to absence of grains and lattice defects; the growth behavior of an amorphous phase and for comparison of pure Fe and nanocrystalline $\mathrm{FeN}$ was studied. Magnetic properties of ferromagnetic films were studied using a DC extraction magnetometer and in order to avoid diamagnetism of the substrate, the magnetic moment was also determined using polarized neutron reflectometry. The results of abovementioned studies are presented and discussed in this article.

\section{EXPERIMENTAL METHODS}

Thin films of $\mathrm{Fe}$ and permalloy $\left(\mathrm{Ni}_{80} \mathrm{Fe}_{20}\right)$ were prepared by magnetron sputtering using a gas mixture of $\mathrm{Ar}+\mathrm{N}_{2}$. The nitrogen partial pressure, defined as, $\mathrm{R}_{N 2}$ $=\mathrm{P}_{N 2} /\left(\mathrm{P}_{N 2}+\mathrm{P}_{A r}\right) \times 100 \%$, was varied at $0,2,5,10,20$, $33,50,83$ and $100 \%$ for Fe and 0, 5, 10, 20, 33, 50, 59, 83 and $100 \%$ for NiFe. The gas flows in the vacuum chamber were controlled using mass flow controllers and the total gas flow for sputtering was kept fixed at $10 \mathrm{~cm}^{3} / \mathrm{min}$. Circular targets of pure Fe or permalloy, $75 \mathrm{~mm}$ in diameter, were sputtered with the gas mixture. A constant sputtering power of $50 \mathrm{~W}$ was used in all depositions. The targets were covered with slits of width $80 \mathrm{~mm}$ to restrict the plasma. The cathode (target) and the substrate were mounted parallel to each other at a distance of about 8 $\mathrm{cm}$. Before depositions a base vacuum of the order of $1 \times 10^{-6}$ mbar was obtained and the vacuum chamber was flushed with $\mathrm{Ar}$ and $\mathrm{N}_{2}$ gas so as to avoid contamination of other gases inside the vacuum chamber. The pressure during deposition was in the range of $4-8 \times 10^{-3}$ mbar. The substrates were mounted below the targets and oscillated with respect to the central position of the target for better uniformity of the deposited samples. All the samples were deposited at room temperature $(\sim 298 \mathrm{~K}$, without intentional heating) on float glass or $\mathrm{Si}(100)$ substrates. Thin films for growth studies were deposited in a single sputtering run (for one composition) onto a glass substrate. The targets were covered with a small slit of size $15 \mathrm{~mm}$ and the substrate was exposed to the center of target for different amount of time to obtain different thicknesses. The substrate was translated using a computer controlled linear translation stage. The thicknesses of the films were determined using x-ray reflectivity (XRR) technique and the structure of the films was investigated using grazing-incidence $\mathrm{x}$-ray diffraction (XRD) using $\mathrm{Cu} \mathrm{K} \alpha$ x-rays. For all the measurements the incident angle was kept fixed just above the critical angle of the film, to minimize the background due to diffraction of the substrate. The bulk magnetic measurements were performed using a DC extraction magnetometer with the magnetic field applied parallel to the plane of the film using a physical property measurement system (PPMS). In order to determine the magnetic moment of the films, independent of substrate magnetism or sample area, the polarized neutron reflectivity (PNR) measurements were performed at the saturation field of the samples. The measurements were performed at fixed angle of incidence in the time of flight (ToF) mode at AMOR(SINQ/PSI) 35

\section{RESULTS AND DISCUSSION}

\section{A. Structural properties : FeN}

Fig. 11 shows the grazing-incidence XRD pattern of Fe films prepared with different nitrogen partial pressure. The film prepared with Ar gas only, shows reflections corresponding to bcc $\alpha$-Fe with orientation in the direction of (110) plane. For the films prepared with $2 \%-20 \%$ nitrogen partial pressure (rest Ar), the XRD pattern shows a structure similar to bcc $\alpha$-Fe, however all the peaks were broad and the peak positions were shifted to lower angle side as compared to the XRD pattern of pure Fe film. The line width of the diffracted pattern can be used to calculate the grain size of the diffracting specimen in the direction perpendicular to the plane of the film using Scherrer formula, ${ }^{36} t=0.9 \lambda / b \cos \theta$, where $t$ is the grain size, $b$ is an angular width in terms of $2 \theta, \theta$ is the Bragg angle and $\lambda$ is the wavelength of the radiation used. For the film prepared with Ar gas only, the average grain size was $13 \pm 1 \mathrm{~nm}$ while in the presence of $2 \%$ nitrogen partial pressure during sputtering, it decreases to $6 \pm 1 \mathrm{~nm}$; half of the value found without any nitrogen. This result indicates that even the presence of nitrogen as small as $2 \%$, significantly affects the growth of Fe crystals and restricts the long-range ordering. The positions of Bragg peaks for the sample prepared with $2 \%$ nitrogen, were shifted to lower-angle side, indicating an increase in the inter-atomic spacing. The average interatomic distance can be estimated using the relation $a=1.23 / 2$ $\sin \theta$, where $\theta$ is taken to be the angle at the center of the peak, and the factor 1.23 is a geometric factor which rationalizes the nearest neighbor distance with the spacing between "pseudo-close packed planes" 37 Comparing the interatomic spacing for the film prepared without ni- 


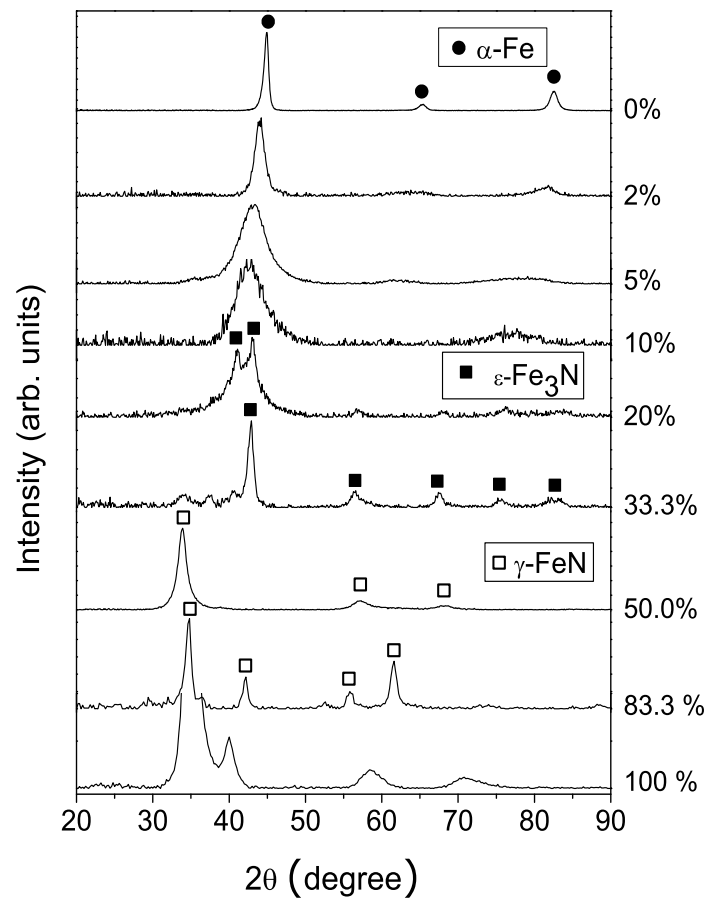

FIG. 1: Grazing incidence x-ray diffraction pattern of FeN thin films prepared with different nitrogen partial pressure.

trogen and with $2 \%$ nitrogen, the average interatomic spacing was found to be expanded by $2 \%$. On increasing the nitrogen partial pressure the width of the reflections further increases and peak position continues to shift towards the lower angle side. At 5 and $10 \%$ nitrogen partial pressure the line width of the peak becomes as large as $4^{\circ}$, which is close to the value found for conventional iron based amorphous alloys $\underline{\underline{3}}$

The amorphous nature of the film deposited at $\mathrm{R}_{N 2}$ $=10 \%$, was confirmed with transmission electron microscopy (TEM). A thin film of thickness $70 \mathrm{~nm}$ was directly deposited on a carbon coated TEM grid. Fig. 2 shows a representative TEM micrograph along with the electron diffraction pattern. Similar micrographs were observed through out the plane of the film. The micrograph essentially showed a feature less structure and the electron diffraction pattern showed diffuse diffraction ring which confirms the amorphous nature of the film.

Fig. 3] shows a plot of average interatomic distance $a$, as function of increase in the nitrogen partial pressure in the range of $0-20 \%$ for $\mathrm{FeN}$, and $0-33 \%$ for $\mathrm{NiFeN}$. As can be seen from the figure, with an increase in the amount of nitrogen, the interatomic spacing continues to increase. However at $20 \%$ the broad hump overlaps with two sharp peaks. The peak positions of the sharp peaks correspond to hcp $\epsilon-\mathrm{Fe}_{3} \mathrm{~N}$ phase. And the overall structure can be considered as a mixture of amorphous bcc-Fe along with hcp $\epsilon-\mathrm{Fe}_{3} \mathrm{~N}$ phase. At $33.3 \%$ nitrogen partial pressure the structure changes completely and $\epsilon$ -

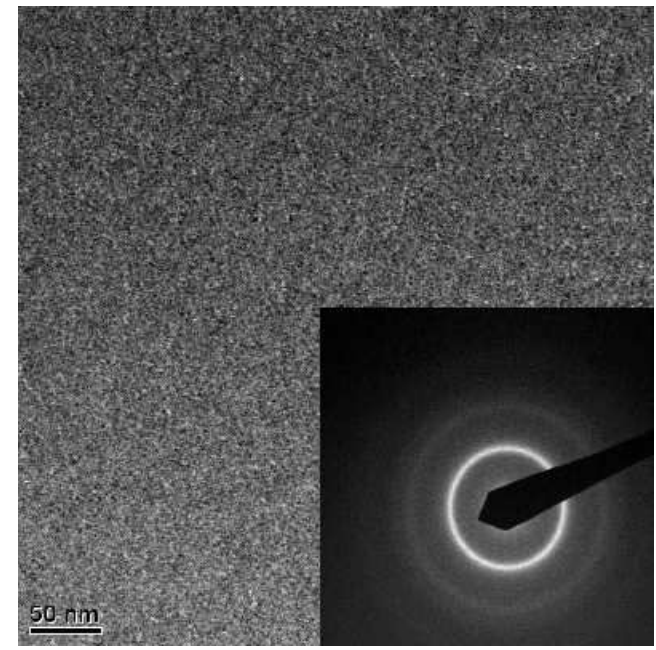

FIG. 2: TEM planar view of the sample prepared at $10 \%$ nitrogen partial pressure. The inset of the picture shows the electron diffraction pattern.

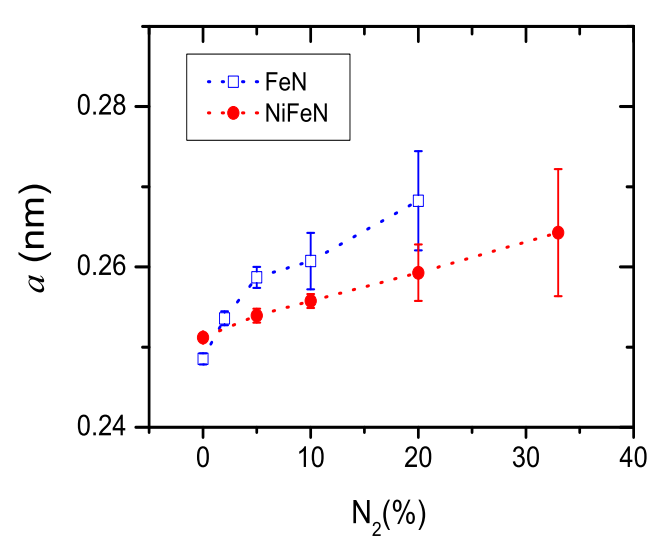

FIG. 3: Inter-atomic spacing as a function of nitrogen partial pressure in FeN and $\mathrm{NiFeN}$.

$\mathrm{Fe}_{3} \mathrm{~N}$ phase along with $\zeta-\mathrm{Fe}_{2} \mathrm{~N}$ phases were obtained. On further increasing the nitrogen partial pressure at $50 \%$, the structure changes again reflections corresponding to new-cubic-type phase were obtained. It may be noted that width of the Bragg peak at $34^{\circ}$ is $\left(1.4^{\circ} \pm 0.01^{\circ}\right)$ corresponding to an average grain size of about $6 \mathrm{~nm}$, which is an indication of formation of a nano grain structure. At $83.3 \%$ nitrogen partial pressure, sharp peaks corresponding to $\gamma^{\prime \prime \prime}$-FeN phase were observed. On further increasing the nitrogen partial pressure to $100 \%$, the peak widths again starts increasing, indicating re-formation a nanocrystalline structure. 


\section{B. Structural properties : NiFeN}

The permalloy target was also sputtered with a mixture of $\mathrm{Ar}+\mathrm{N}_{2}$ by varying the nitrogen partial pressure in the range of $0-100 \%$. Fig. 4 shows grazing incidence x-ray

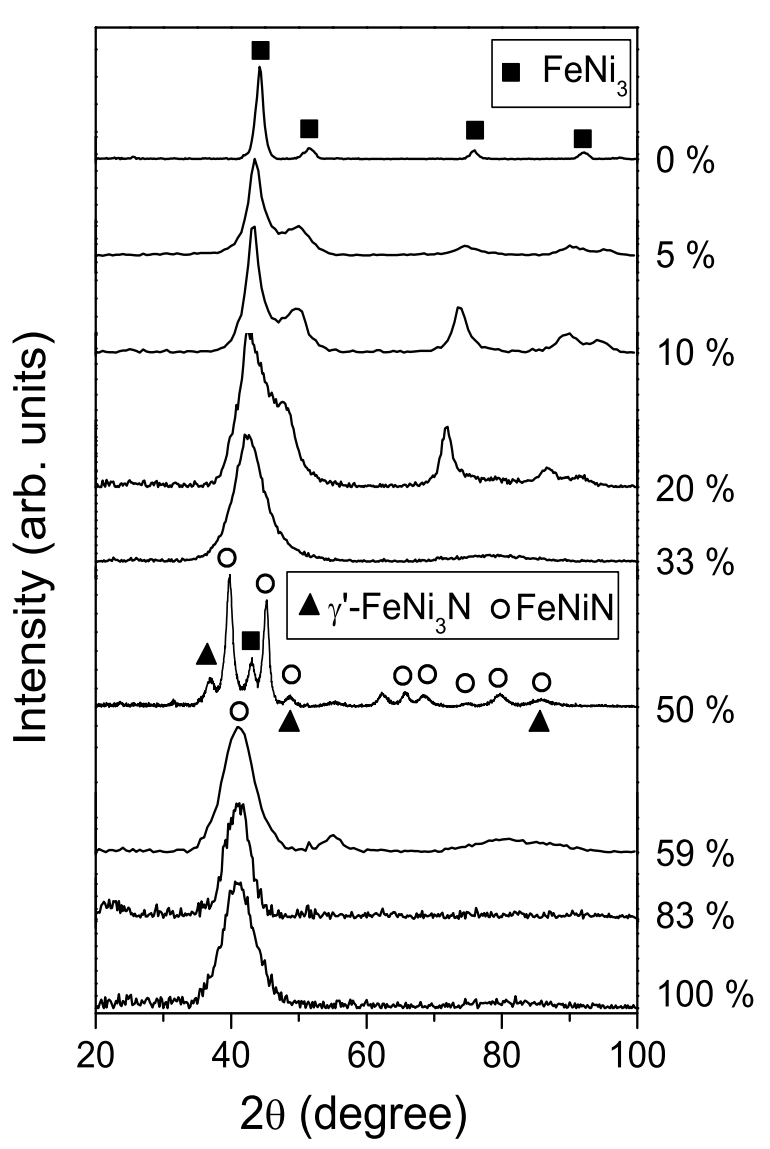

FIG. 4: Grazing incidence x-ray diffraction pattern of $\mathrm{NiFeN}$ thin films prepared with different nitrogen partial pressure.

diffraction pattern of $\mathrm{NiFeN}$ thin films prepared at different nitrogen partial pressure. The film prepared with Ar gas only shows reflections corresponding permalloy phase as indexed in the figure. As the nitrogen partial pressure is increased, the reflection starts broadening and the reflection with indices (111) and (200) starts merging together. A clear shift in the positions of Bragg peak is also evident. The grain size for the film sputtered with Ar only was $7 \mathrm{~nm}$ which decreases to $3.5 \mathrm{~nm}$ after sputtering with 5 or $10 \%$ nitrogen. With a further increase in the nitrogen partial pressure, an amorphous phase appeared at $33 \%$. As compared to $\mathrm{FeN}$, the shifts in the positions of Bragg peaks were rather small (see fig. 3). Also overall increase in the interatomic distance at similar nitrogen pressure was smaller in $\mathrm{NiFe}$ as compared to Fe. A discussion related to this issue is given in section 3 .
While comparing the observed results with that of $\mathrm{NiN}$ studied by Kawamura et al.,34 similar broadening and expansion of unit cell of $\mathrm{Ni}$ was observed. On increasing the $\mathrm{R}_{N 2}$ to $50 \%$ with $\mathrm{NiFe}$, the structure was changed completely and several peaks were observed in the XRD pattern. The phase identified at this pressure is a mixture of FeNiN $+\gamma^{\prime}-\mathrm{FeNi}_{3} \mathrm{~N}$. On further increasing $\mathrm{R}_{N 2}$ to $59 \%$ or above, a broad hump around $2 \theta=40^{\circ}$ appears along with faint reflections at higher angles. This hump appears to be an envelop of several reflection observed for $\mathrm{R}_{N 2}=50 \%$ sample and indicates re-amorphization of the polycrystalline permalloy nitride structures formed at $\mathrm{R}_{N 2}=50 \%$.

Nanocrystallization or amorphization induced by reactive nitrogen sputtering in $\mathrm{Fe}, \mathrm{Ni}$ and $\mathrm{NiFe}$ can be explained with a single mechanism. At low nitrogen partial pressures, nitrogen ions does not react with $\mathrm{Fe}, \mathrm{Ni}$ or NiFe and nitrogen is incorporated in the interstitial sites, making an expansion of the unit cell. At intermediate nitrogen pressure, a chemical reaction between nitrogen and $\mathrm{Fe}, \mathrm{Ni}$ or $\mathrm{NiFe}$ is favorable which results in formation of nitride phases. At further higher nitrogen pressures, deformation of the formed nitride phase starts and the end structure is again nanocrystalline or amorphous. Detailed mechanism inducing nanocrystallization or amorphization is discussed in section 3 .

\section{Crystallization behavior of amorphous films}

From the observed XRD results it is evident that when nitrogen partial pressure during sputtering is 5 and $10 \%$, an amorphous phase of FeN was formed while at $33 \%$ and above $50 \%$, amorphous $\mathrm{NiFeN}$ phase was formed. It would be interesting to study the crystallization behavior of these amorphous phases in order to understand their properties. Two sets of samples were chosen for crystallization studies (i) Fe-rich, FeN samples prepared at $\mathrm{R}_{N 2}=2,5,10$ and $20 \%$ and (ii) N-rich, NiFeN sample prepared at $\mathrm{R}_{N 2}=83 \%$. These films were annealed in a vacuum furnace isochronally for 1 hour at each temperature. In order to avoid the fluctuations in temperature all the four FeN films were annealed simultaneously in the vacuum furnace. Fig. 4 (a-d) shows the grazing incidence XRD pattern of FeN annealed films. It is interesting to observe that the films prepared with 2 and $5 \%$ nitrogen partial pressure were highly unstable, and even at 150 ${ }^{\circ} \mathrm{C}$, Bragg peaks corresponding to $\alpha^{\prime \prime}$-FeN appear in the XRD pattern. The film prepared with $\mathrm{R}_{N 2}=10 \%$, was found to be more stable and it remained amorphous up to an annealing temperature of $200{ }^{\circ} \mathrm{C}$. On further annealing at $300^{\circ} \mathrm{C}$ the amorphous hump splits into three sharp peaks corresponding to $\epsilon-\mathrm{Fe}_{2} \mathrm{~N}_{1-z}$ phase and at $400{ }^{\circ} \mathrm{C}$, new peaks corresponding to $\alpha^{\prime \prime}-\mathrm{Fe}_{16} \mathrm{~N}_{2}$ phase were observed. For the film prepared with $\mathrm{R}_{N 2}=20 \%$, the XRD pattern reveals a composite structure consisting of amorphous bcc-Fe and $\epsilon$-Fe ${ }_{2} \mathrm{~N}_{1-z}$ phase in the as-deposited state and after annealing at $150{ }^{\circ} \mathrm{C}$, the structure crys- 

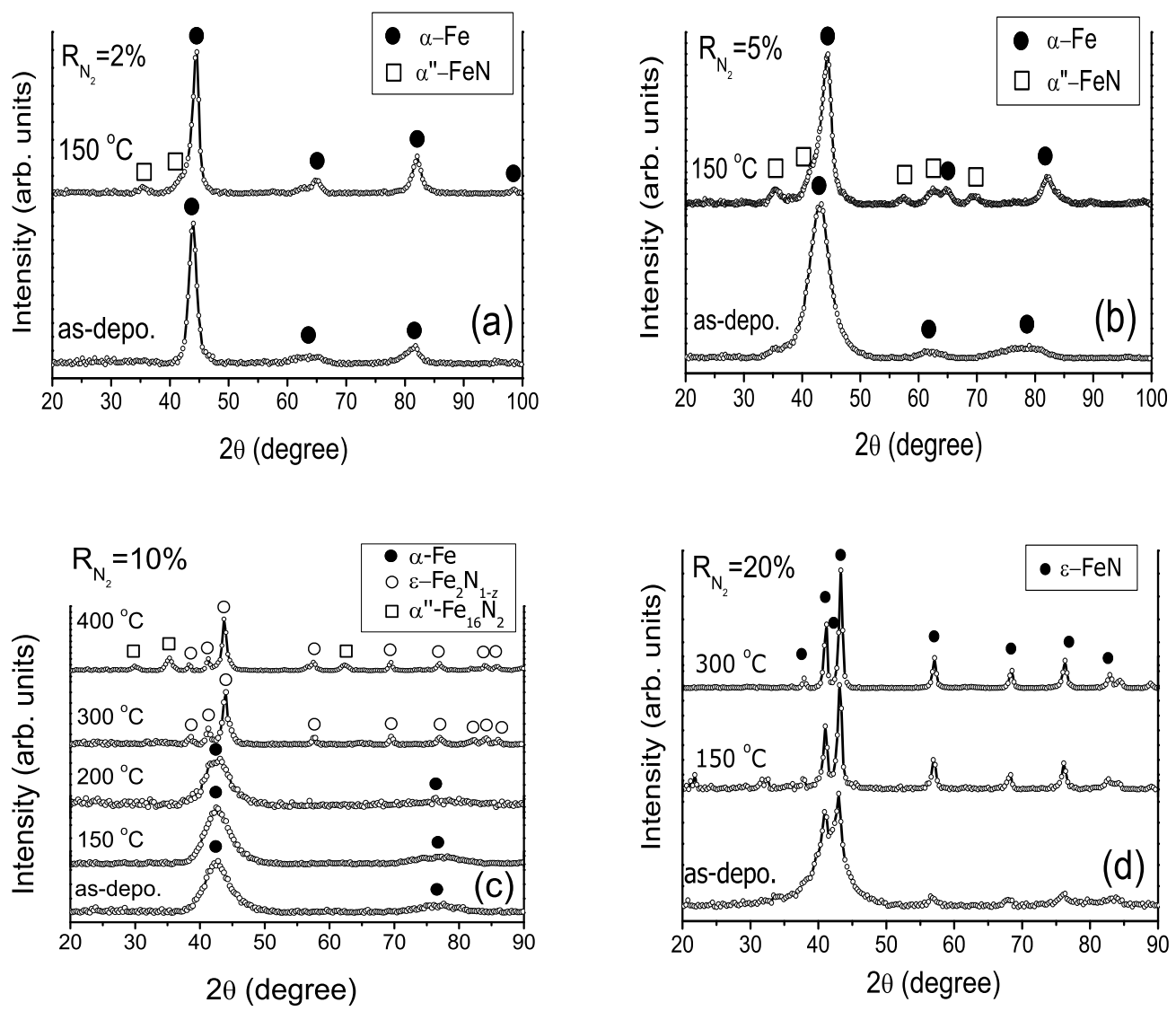

FIG. 5: Grazing incidence x-ray diffraction pattern of FeN film prepared with 2\% (a), $5 \%$ (b), $10 \%$ (c) and $20 \%$ (d), in the as-deposited state and after vacuum annealing at various temperatures. The films were annealed isochronally for 1 hour.

tallizes into $\epsilon-\mathrm{Fe}_{2} \mathrm{~N}_{1-z}$ phase. Observed crystallization behavior of amorphous phase shows that the amorphous phase shows a better stability for the film prepared with $\mathrm{R}_{N 2}=10 \%$, and the films prepared either with higher or lower nitrogen are formed in a highly metastable state. Fig. [6] shows grazing incidence XRD pattern of the NiFeN film prepared at $\mathrm{R}_{N 2}=83 \%$, after annealing at different temperatures. As can be seen from the figure, up to an annealing temperature of $250{ }^{\circ} \mathrm{C}$, no significant changes in the XRD pattern were observed. While after annealing at $350{ }^{\circ} \mathrm{C}$, several peaks were observed. The most intense peaks corresponds to $(\mathrm{FeNi})_{3} \mathrm{~N}$ and $\gamma^{\prime}-\mathrm{FeNi}_{3} \mathrm{~N}$ phases. Smaller peaks at 53.6, 58.2 and $77.8^{\circ}$ could not be identified. After further annealing at $500^{\circ} \mathrm{C}$, no nitride phase was observed, indicating out diffusion of nitrogen.

The observed crystallization behavior of both amorphous FeN and NiFeN phases is different as compared to iron based binary or multi-component alloys. In conventional metal-metal amorphous alloys, generally crystallization occurs in 2 steps, in the first step a nanocrystalline microstructure co-exists with parent amorphous phase whereas in the second step an intermetallic compound along with nanocrystalline phase precipitates out. The nominal reaction for such crystallization process had been given as: amorphous $\rightarrow \alpha+$ amorphous $\rightarrow \alpha+$ $\beta$; where $\alpha$ is the primary phase that precipitates out from the amorphous matrix and $\beta$ is an intermetallic compound ${ }^{38.39}$ In the present case, however, crystallization takes place in a single step and annealing at temperatures above crystallization temperatures essentially results in nitrogen out diffusion. The amorphous structure remained amorphous up to certain annealing temperature and thereafter mixed nitride phase were observed. On further annealing due to nitrogen out diffusion pure metallic or nitrogen poor phases are obtained.

\section{Surface properties and growth behavior of FeN films}

The thickness of $\mathrm{FeN}$ and $\mathrm{NiFeN}$ thin films deposited for $\mathrm{R}_{N 2}=0-100 \%$, were determined using x-ray reflectivity technique. Since both FeN and NiFeN thin films were deposited in a similar manner, detailed surface and growth behavior of $\mathrm{FeN}$ thin films was investigated. Fig. [7] shows x-ray reflectivity pattern of $\mathrm{FeN}$ thin films prepared at different nitrogen partial pressures. The x-ray reflectivity pattern was fitted using a computer 


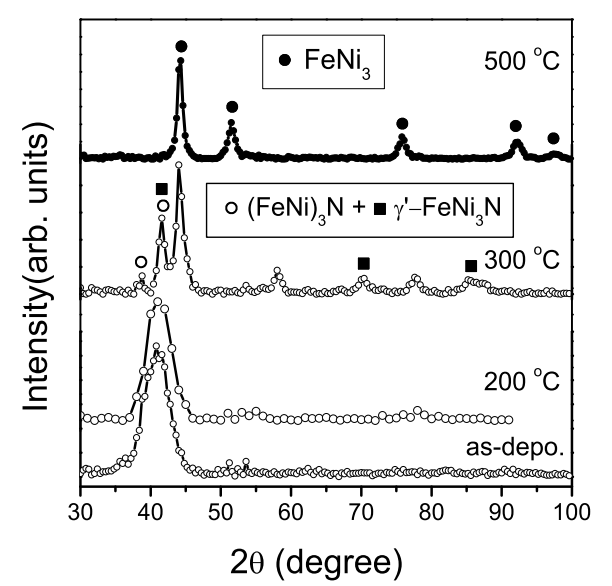

FIG. 6: Grazing incidence x-ray diffraction pattern of NiFeN thin film prepared with $83 \%$ nitrogen partial pressure after annealing at different temperatures.

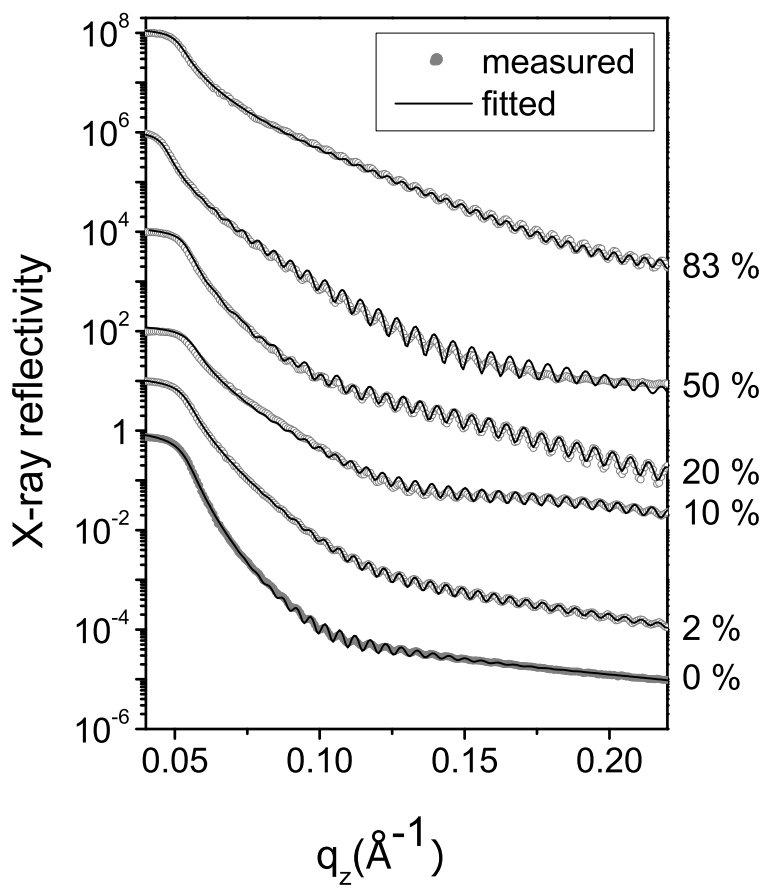

FIG. 7: X-ray reflectivity pattern of FeN thin films prepared with different nitrogen partial pressure.

program ${ }^{40}$ based on Parratt's formalism $\underline{41}$ Oscillations due to total thickness of the films can be clearly seen in the reflectivity pattern. The thickness of the films obtained after fitting the pattern and was found in the range of $90-100 \mathrm{~nm}$. The fitted parameters are given in table 【 A detailed fitting of the pattern revealed that a
TABLE I: Fitted x-ray reflectivity parameters for FeN thin films prepared at different nitrogen partial pressures.

\begin{tabular}{cccc}
\hline \hline$R_{N 2}$ & $\begin{array}{c}\text { film } \\
\text { thickness } \\
\mathrm{nm}( \pm 0.2)\end{array}$ & $\begin{array}{c}\text { film } \\
\text { roughness } \\
\mathrm{nm}( \pm 0.1)\end{array}$ & $\begin{array}{c}\text { sub } \\
\text { roughness } \\
\mathrm{nm}( \pm 0.2)\end{array}$ \\
\hline 0 & 103.5 & 3.3 & 0.6 \\
2 & 105.4 & 2.0 & 0.6 \\
10 & 101.6 & 0.4 & 0.6 \\
20 & 98.3 & 1.0 & 0.6 \\
50 & 93.5 & 1.0 & 0.6 \\
83 & 113.5 & 0.8 & 0.6 \\
\hline \hline
\end{tabular}

thin layer with density about $50 \%$ of the bulk of the layers is formed on the surface. Such a layer may be formed due to "oxidation" of the surface when exposed to atmosphere. The thickness of this layer was typically $2-3 \mathrm{~nm}$. It is interesting to see that the roughness of the film prepared with Ar gas only, was $3.3 \mathrm{~nm}$, which decreases to 2 $\mathrm{nm}$ at $\mathrm{R}_{N 2}=2 \%$, and was only $0.4 \mathrm{~nm}$ for $\mathrm{R}_{N 2}=10 \%$. At $\mathrm{R}_{N 2}=20$ and $50 \%$ the roughness again increases to $1 \mathrm{~nm}$ and at $83 \%$ again it decreases slightly. While looking at microstructure of the deposited film obtained from XRD measurements, there is a clear indication that an amorphous phase is formed at lower and higher nitrogen partial pressures. A decrease in the roughness of film is not unexpected since amorphous structure is free of grains and lattice defects, which may result in formation of a smoother surface or interface. With the observed decrease in the roughness of the film for amorphous samples, it would be interesting to study the growth behavior of these films. For this purpose a series of FeN thin films were deposited at $\mathrm{R}_{N 2}=0,10$ and $83 \%$. In order to minimize the parameters influencing the growth of a thin film, all the film with one composition were prepared in a single sputtering run. For this purpose, the area of sputtering target was masked with a small slit of size $15 \mathrm{~mm}$ and all the films were prepared on a glass substrate by exposing the substrate for different times to the plasma at different positions on the substrate to obtain different thicknesses.

All the films were prepared in the thickness range of 10$150 \mathrm{~nm}$. X-ray reflectivity measurements on all the films were performed in specular and off-specular mode. The off-set in off-specular measurements was taken at the onset of specular reflection peak in the rocking scan. This offset was $0.05^{\circ}$. The off-specular data was subtracted from the specular data to obtain the "true-specular" data. Fig. 8(a-c) shows the XRR patterns of abovementioned thin films. The patterns were fitted using a procedure as describe earlier. It was observed that for pure iron film, the surface roughness increases monotonically with an increase in the thickness while for the amorphous FeN film the roughness of the film increases at a very slow rate. For the case when $\mathrm{FeN}$ forms a nanocrystalline nitride at $\mathrm{R}_{N 2}=83 \%$, the surface roughness again show an increase with the thickness. Previous studies have 



FIG. 8: X-ray reflectivity pattern of pure Fe film (a), FeN film with $10 \% \mathrm{~N}$ (b) and FeN film with $83 \%$ nitrogen partial pressure (c). The evolution of film roughness with film thickness (d). 
shown that the rms roughness $(\sigma)$ exhibits a power-law behavior $\stackrel{20.42}{2}$ as a function of the film thickness, given as: $\sigma \sim \mathrm{t}^{\beta}$, accordingly, a double logarithmic plot of the rms roughness of the films versus the film thickness should yield a linear relation. Fig. 8 (d) shows a plot rms roughness versus film thickness for the abovementioned samples and a straight line fit to the data yields the roughness growth exponent, $\beta$. For pure Fe film $\beta=$ 0.35 , for amorphous FeN film prepared at $\mathrm{R}_{N 2}=10 \%$, $\beta=0.14$, and for nanocrystalline $\mathrm{FeN}$ film prepared at $\mathrm{R}_{N 2}=83 \%, \beta=0.39$. The value of $\beta$ for amorphous FeN is very close to that obtained for amorphous $\mathrm{SiO}_{2}{ }^{43}$ and nitrogen rich amorphous $\mathrm{FeN}$ prepared using ion-beam sputtering ${ }^{20}$ Under the various growth models described in literature, it may be observed that for the values of the roughness growth exponent lying in the range of 0.1-0.25, the growth can be well described by the KPZ model, first introduced by Kardar, Parisi, and Zhang 44 This type of the growth processes takes into account a random deposition and a limited relaxation of the particles at the surface. For the present case the value of for amorphous sample lies well in this range, while for both pure $\mathrm{Fe}$ and nanocrystalline $\mathrm{FeN}$, the value of $\beta$ is higher. A higher value of $\beta$ indicates non KPZ type growth which is often observed for polycrystalline elements. The obtained results clearly show a different growth mechanism for amorphous film and support the argument that amorphous films yield smoother surfaces. It may be noted that the absolute roughnesses of the films prepared with smaller slits $(15 \mathrm{~mm})$ in front of the targets were smaller as compared with the larger slits $(80 \mathrm{~mm})$.

\section{E. Bulk magnetization measurements of FeN and NiFeN films}

Fig. 9] shows MH curve of the FeN samples prepared at different nitrogen partial pressure. For the film prepared with $\mathrm{Ar}$ gas only, the behavior is as-expected for iron, and the value of saturation magnetization $(\mathrm{Ms})$ was almost equal to reported values for bulk Fe. For the sample prepared at $\mathrm{R}_{N 2}=2 \%$, the $\mathrm{MH}$ loop shape changes to a typical soft-magnetic. While the value of saturation magnetization is almost equal to pure $\mathrm{Fe}$, the $\mathrm{MH}$ curve shows a significant decrease in the value of coercivity $(\mathrm{Hc})$. It may be noted that the average grain size (D) for pure Fe is about $13 \mathrm{~nm}$, while for the sample prepared with $2 \%$ nitrogen partial pressure, the grain size reduce to 6 $\mathrm{nm}$. A decrease in Hc in nanocrystalline ferromagnets is expected as envisaged in the random-anisotropy model (RAM) $\stackrel{45.46 .47}{=}$ It may be noted that the ferromagnetic exchange length $\left(\mathrm{L}_{e x}\right)$ for $\alpha$-Fe is $15-23 \mathrm{~nm} \cdot{ }^{48.49}$ For grain sizes $\mathrm{D}<\mathrm{L}_{e x}, \mathrm{Hc}$ decreases with a decrease in grain size by $\mathrm{D}^{6}$. A reduction in grain size below ferromagnetic exchange length allows exchange coupling between the neighboring grains and results in a reduced effective anisotropy $\langle K\rangle$. In the present case, however, the decrease in Hc was not found to vary with $\mathrm{D}^{6}$, rather it fol-

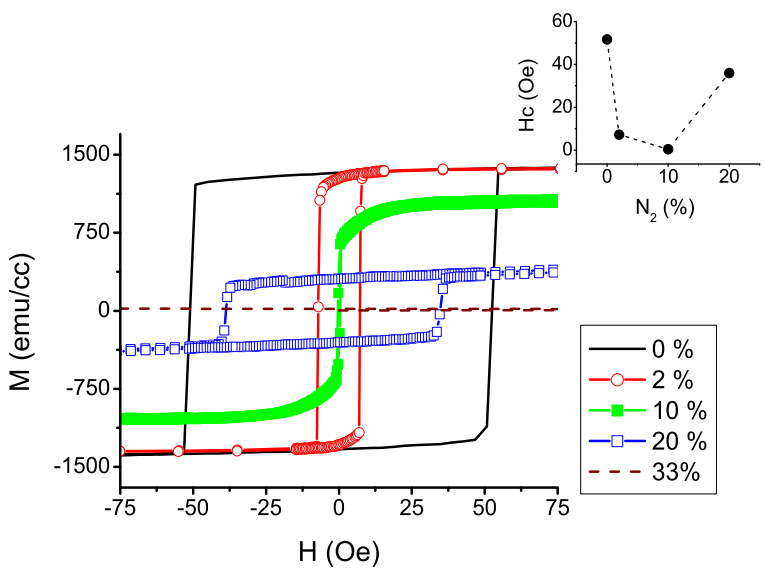

FIG. 9: Magnetization measurements of FeN thin films prepared at different nitrogen partial pressures. The inset shows a variation in coercivity as a function of nitrogen partial pressure during sputtering.

lows $\mathrm{D}^{2-3}$ type behavior. Since in the present case thickness of the films is small, it is likely that effective averaging would be only in the plane (area) of the film. In this situation the ferromagnetic correlation volume would be proportional to $\mathrm{L}_{e x}{ }^{2}$ only, in contrast to $\mathrm{L}_{e x}{ }^{3}$, in case of bulk materials. This would reduce the number of grains over which the averaging is done, and therefore a reduction in the magnetic anisotropy and $\mathrm{Hc}$ is not expected to vary as $\mathrm{D}^{6}$ as observed in nanocrystalline ribbons or powders. Assuming averaging over $\mathrm{N}=\left(\mathrm{L}_{e x} / \mathrm{D}\right)^{2}$ number of grains, the effective anisotropy would be:

$$
\langle K\rangle=\frac{K_{1}^{2} D^{2}}{A},
$$

where $\mathrm{K}_{1}$ is magneto-crystalline anisotropy of the grains and $\mathrm{A}$ is the exchange stiffness. This would mean that in case of a thin film, $\mathrm{Hc}$ would follow $\mathrm{D}^{2}$ type behavior rather than $\mathrm{D}^{6}$, as pointed out by Hoffmann et al ${ }^{50}$ The observed decrease in $\mathrm{Hc}$, for the sample prepared in presence of $2 \%$ nitrogen partial pressure can be understood accordingly.

On the other hand when nitrogen partial pressure was increased to $10 \%$, the alloy forms an amorphous structure and the magnetic measurements show a decrease in Ms as well as Hc. A decrease in Hc can be understood within RAM, when averaging is done on very fine grains and magnetization follow the easy direction of each individual grain. The decrease in the value of Ms can be understood due to weakening of the exchange coupling between the grains. Further, at $\mathrm{R}_{N 2}=20 \%$, the Hc increase abruptly while Ms continues to decrease.

It may be noted that at this nitrogen partial pressure amorphous phase co-exists with hcp- $\epsilon-\mathrm{Fe}_{3} \mathrm{~N}$ phase. In case $\epsilon-\mathrm{Fe}_{3} \mathrm{~N}$ phase is nonmagnetic (as found at higher $\mathrm{R}_{N 2}$ ), presence of a nonmagnetic phase among the ferromagnetic fine grains would result in a decrease in 
exchange length, which in accordance with the RAM, causes anisotropy and coercivity to increase because of incomplete averaging-out of random anisotropies of grains within the exchange volume. The observed increase in the Hc and decrease in Ms can be understood with this argument. At further higher nitrogen partial pressure where $\epsilon-\mathrm{Fe}_{3} \mathrm{~N}$ phase along with $\zeta-\mathrm{Fe}_{2} \mathrm{~N}$ phases were obtained, the magnetization was almost zero, indicating nonmagnetic nature of the film at this nitrogen pressure. The films deposited at even higher $\mathrm{R}_{N 2}$ were also nonmagnetic.

Crystallization behavior of the amorphous film deposited at $\mathrm{R}_{N 2}=10 \%$, was also studied with magnetization measurements of the samples annealed at different temperatures. Fig. 10] shows magnetization measure-

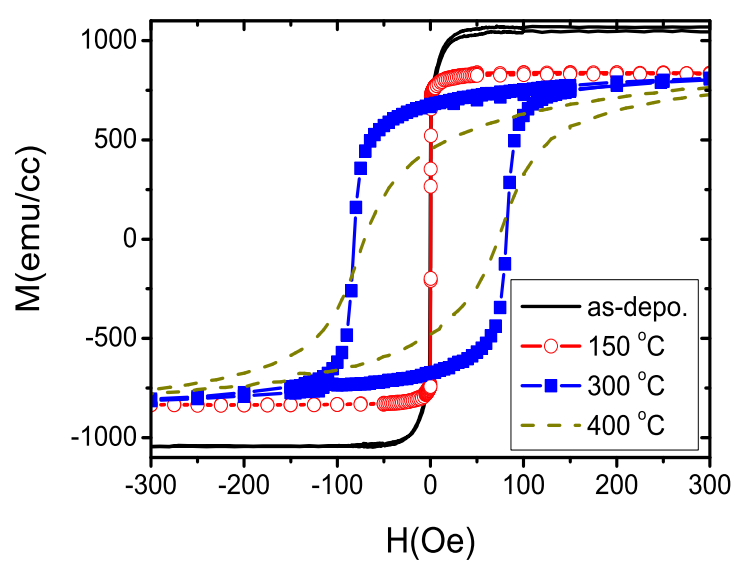

FIG. 10: Magnetization measurements of FeN thin film prepared at $10 \%$ nitrogen partial pressure as function of annealing temperature.

ments after annealing at different temperatures as discussed in the previous section. The M-H loop shape up, before crystallization temperature was similar to that in the as-deposited state. The only appreciable change was the shape of the $\mathrm{M}-\mathrm{H}$ loop, which became more square after annealing. Such a change in the M-H loop shape is directly related to removal of strains which might have developed during the deposition. At higher annealing temperature where crystallization of amorphous phase takes place, the loop shape was completely different. There was a sharp increase in the value of coercivity and the average value of Ms decreased. At further higher temperature, the value of Ms further decreased and the loop shape looks broader. From the XRD measurements it is evident that upon crystallization $\epsilon-\mathrm{Fe}_{3} \mathrm{~N}$ phase precipitates out and an increase in the Hc and decrease in Ms is similar as observed for the sample prepared at $\mathrm{R}_{N 2}=$ $20 \%$, where $\epsilon-\mathrm{Fe}_{3} \mathrm{~N}$ phase existed. The observed magnetization behavior can be understood accordingly.

The magnetization measurements were also carried out

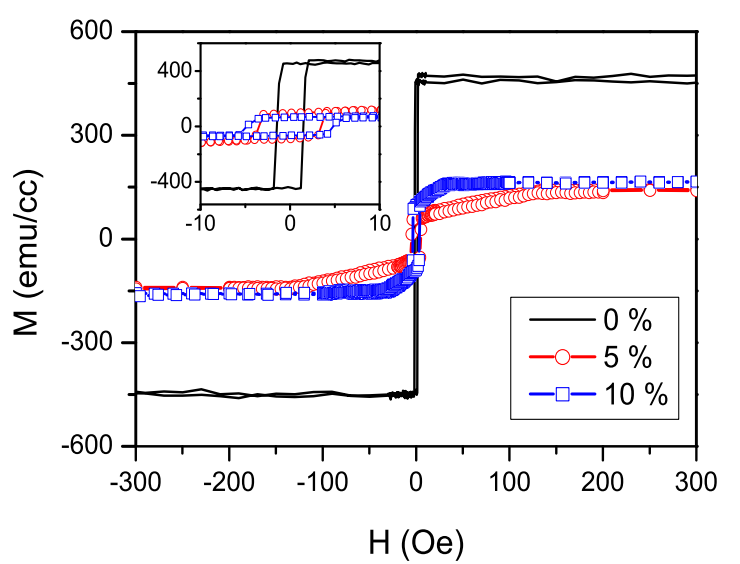

FIG. 11: Magnetization measurements of NiFeN thin films prepared at different nitrogen partial pressures.

in $\mathrm{NiFeN}$ films as a function nitrogen partial pressure during sputtering. The $\mathrm{M}-\mathrm{H}$ loop for the sample prepared with $\mathrm{Ar}$ gas only is matching well with the values obtained for permalloy 51 For the samples prepared with increased $\mathrm{R}_{N 2}$, the magnetization decreases rapidly and the values of coercivity increased (see Fig. 11). As will be discussed later, the start structure with NiFe is fcc, and the volume of interstitial is much larger in case of fcc as compared with a bcc Fe. This allows more nitrogen atoms to be incorporated within the unit cell of fcc $\mathrm{NiFe}$, which eventually results in decrease in magnetization more rapidly as compared with bcc Fe. The observed magnetization behavior may be understood with this argument.

\section{F. Polarized neutron reflectivity measurements}

In the present case since the samples were deposited either on glass or Si substrate and the thickness of the deposited films was in the range of $100 \mathrm{~nm}$, the diamagnetism of the substrates might results in erroneous values of absolute saturation magnetization when measured with DC extraction magnetometer. Also the errors in determining the size of the measured samples may lead to further errors in the values of absolute magnetization. With these two parameters in mind, the magnetization of the FeN samples was also determined using Polarized neutron reflectivity (PNR). PNR is a technique which is able to yield the absolute value of magnetic moment per atom in a magnetic thin film with high accuracy 52 In contrast to bulk magnetization magnetometer technique (e.g. DC extraction, VSM or SQUID), no correction due to magnetic signal from the substrate has to be applied in PNR. Further, the samples dimensions and mass does not play a crucial role in determination of magnetic mo- 
ment. During the experiment, polarized neutrons with spin parallel or antiparallel to the direction of magnetization on the sample are reflected-off the surface of the sample at grazing incidence. The measurements were performed with an applied field of 400 Oe, which is sufficient to reach the saturation magnetization in all the samples. The measurements were carried out in the ToF mode at a fixed angle of incidence. The ToF-PNR has an advantage as during the measurement of spin up and spin down reflectivities, only the polarization of the incoming beam is changed by switching the direction of the applied field at the polarizing supermirror ${ }^{35}$ No movement of the sample is required as often done in $\theta-2 \theta$ mode. The potential energy of a neutron in the $i^{\text {th }}$ region of the sample is given by: 52.53

$$
V_{i}=\frac{2 \pi \hbar^{2}}{m_{n}} \rho_{i} b_{i}+\mu_{n} \bullet B_{i}
$$

where $\mathrm{m}_{n}, \rho_{i}, \mathrm{~b}_{i}, \mu_{n}, \mathrm{~B}_{i}$ are mass of neutron, atomic density, coherent scattering length, neutron moment and magnetic field. This potential gives rise to spin dependent reflectivity for the cases when incident polarization is parallel to the direction of magnetization in the sample $(+)$ or antiparallel (-). Fig. 12 shows PNR data on the

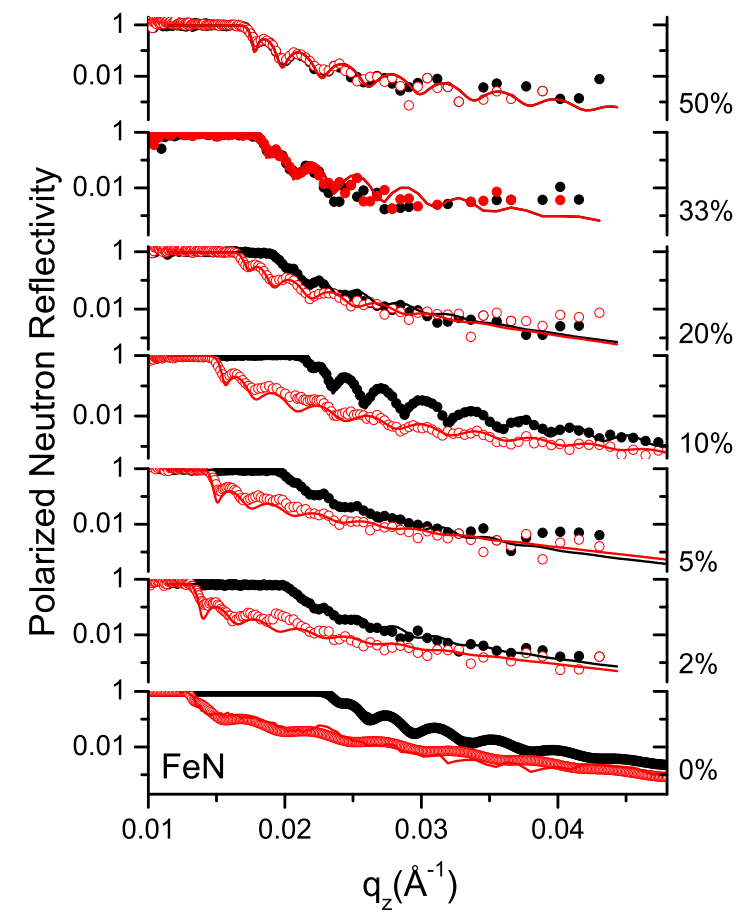

FIG. 12: Polarized neutron reflectivity measurements of FeN thin films prepared at different nitrogen partial pressures. Closed circles represents the data corresponding to spin up reflectivity $R+$ and open circles to spin down reflectivity $R-$. The solid line represents fitting to the experimental data.

samples prepared as function increased nitrogen partial

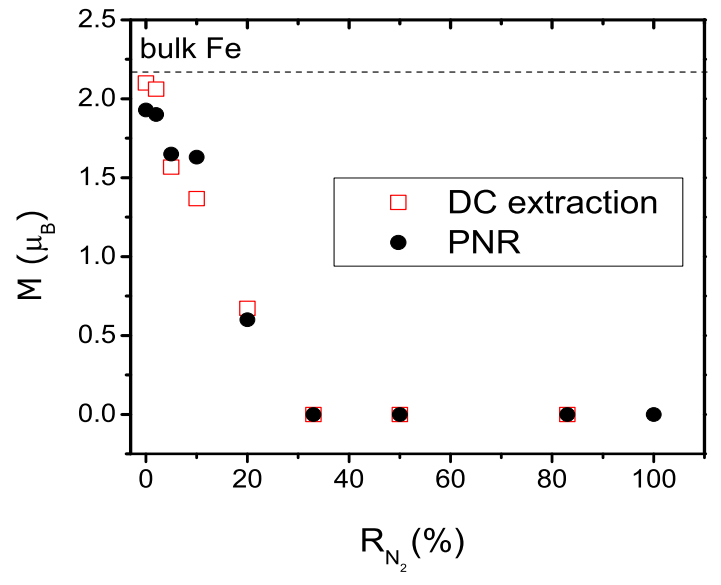

FIG. 13: Magnetic moment as obtained with the bulk magnetization measurements and polarized neutron reflectivity.

pressure during sputtering. As the amount of nitrogen partial pressure is increased, the edge in $R$ - shows a shift towards higher $\mathrm{q}_{z}$ values, and the separation between $R+$ and $R$ - reflectivities decreases continuously. Finally for $\mathrm{R}_{N 2}>20$, both $R+$ and $R$ - are merged together, indicating that the sample has became nonmagnetic. The magnetic moment in each case was determined by fitting the experimental data using a computer program. ${ }^{54}$ Fig. 13 shows a plot of obtained values of magnetic moment with PNR and bulk magnetization measurements. Perusal of the figures gives a clear indication that the values of magnetic moment obtained with the two techniques lies in the same range within the experimental errors. It is interesting to note that at no point the magnetic moment is higher than that of pure.

\section{MECHANISM INDUCING NANOCRYSTALLIZATION OR AMORPHIZATION}

From the results and discussion given in the previous section, it is clear that both $\mathrm{Fe}$ and NiFe form a nanocrystalline or amorphous structures when sputtered with reactive nitrogen. Only at some specific nitrogen partial pressures, polycrystalline structures are obtained. The mechanism inducing nanocrystallization or amorphization can be understood in terms of incorporation of nitrogen within the crystal structures of Fe and NiFe and rapid quenching of adatoms at the substrate. Atoms with low $\mathrm{Z}$ e.g. B, C or $\mathrm{N}$ can easily occupy interstitial sites causing an expansion of the unit cell as well as restricting the long range ordering. It may be noted that for Fe the structure is bcc, while for NiFe, it is fcc. The probability of occupying the interstitial sites in the bcc and fcc crystal system is different because of different close packing. 
In bcc-Fe the atoms may occupy the tetrahedral inter-
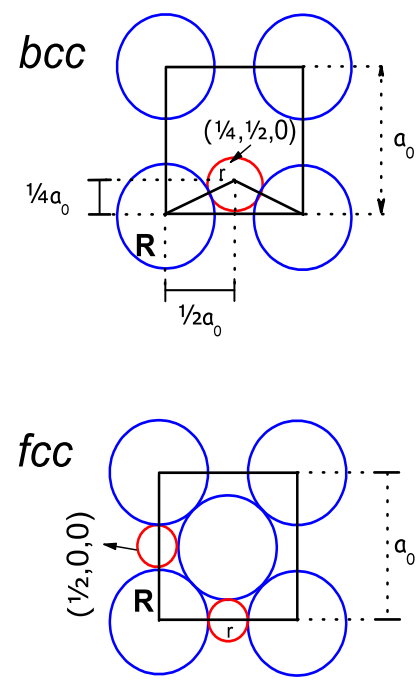

FIG. 14: Distribution of interstitial site in bcc and fcc structures.

stitial sites (see Fig. 14), such as $\left(\frac{1}{4}, \frac{1}{2}, 0\right)$, while in a fcc structure, nitrogen atom may be located either at the octahedral sites at the edge center of unit cell $\left(\frac{1}{2}, 0,0\right)$ and/or at the center of the unit cell $\left(\frac{1}{2}, \frac{1}{2}, \frac{1}{2}\right)$. The probability of occupation of nitrogen atoms at the interstitial sites in the two structures can be obtained by calculating the size of the interstitials for the two cases:

The radius of tetrahedral interstitial site at $\left(\frac{1}{4}, \frac{1}{2}, 0\right)$ location in bcc-Fe can be written as:

$$
r_{i n t}=\left[\left(\frac{1}{2} a_{0}\right)^{2}+\left(\frac{1}{4} a_{0}\right)^{2}\right]^{\frac{1}{2}}-R_{b c c},
$$

where $R_{b c c}$ is the radius of bcc Fe and $a_{0} \quad(=0.2866 \mathrm{~nm}$ )is the lattice constant of the bcc Fe atom and $\mathrm{R}_{b c c}=$ $\sqrt{3} \mathrm{a}_{0} / 4=0.1241 \mathrm{~nm}$. From equation (3) the radius of $\left(\frac{1}{4}, \frac{1}{2}, 0\right)$ location of interstitial site $\mathrm{r}_{\text {int }}$ for bcc Fe is 0.0361 nm. Similarly we can calculate the radius of interstitial site for fcc $\mathrm{NiFe}$ at the octahedral sites $\left(\frac{1}{2}, 0,0\right)$ using the expression:

$$
2 r_{i n t}=a_{0}-2 R_{f c c}
$$

Where $a_{0}=0.3545 \mathrm{~nm}$ for $\mathrm{NiFe}$ and $\mathrm{R}_{f c c}$ is the radius of fcc NiFe and $\mathrm{R}_{f c c}=\sqrt{2} \mathrm{a}_{0} / 4=0.1253 \mathrm{~nm}$. From equation (4) the radius of $\left(\frac{1}{2}, 0,0\right)$ location of interstitial site $r_{i n t}$ for fcc is $0.0523 \mathrm{~nm}$.
The intestinal site in the bcc Fe is smaller than that in fcc NiFe alloy. Whereas both are smaller than atomic radius of nitrogen atom $(0.075 \mathrm{~nm})$. Therefore for both $\mathrm{Fe}$ and $\mathrm{NiFe}$, nitrogen occupying the interstitial sites would cause a distortion to the unit cell and it is expected that this distortion should be more effective for bcc Fe as compared with fcc NiFe while depositing at a given nitrogen partial pressure. As a matter fact it is clear from our $\mathrm{XRD}$ results that almost complete amorphization of $\mathrm{Fe}$ was observed at $\mathrm{R}_{N 2}=10 \%$, while incase of NiFe, fully amorphous state was obtained at $\mathrm{R}_{N 2}=33 \%$. This result clearly indicated that nitrogen atoms gradually occupy the interstitial space within bcc or fcc structure and since available space in a fcc structure is larger, fcc structure allowed more nitrogen atoms to be incorporated. Further, when nitrogen partial pressure was increased beyond the one at which final amorphous structure was obtained, the structure of both $\mathrm{Fe}$ and $\mathrm{NiFe}$ was changed basically to $\mathrm{A}_{3} \mathrm{~N}(\mathrm{~A}=\mathrm{Fe}$ or $\mathrm{NiFe})$.

From the energetics of binary iron nitrides 55 (at room temperature), it may be noted that the heat of formation for $\epsilon-\mathrm{Fe}_{3} \mathrm{~N}_{x}$ is lower $\left(-40\right.$ to $\left.-45 \mathrm{~kJ} \mathrm{~mol}^{-1}\right)$ as compared with neighboring e.g. $\mathrm{Fe}_{4} \mathrm{~N}\left(-12 \mathrm{~kJ} \mathrm{~mol}^{-1}\right)$ or $\mathrm{Fe}_{2} \mathrm{~N}(-34$ $\mathrm{kJ} \mathrm{mol}^{-1}$ ) phases. On the other hand nitrogen richest phase $\gamma^{\prime \prime \prime}-\mathrm{FeN}_{0.91}$ has the lowest enthalpy of formation $\left(-47 \mathrm{~kJ} \mathrm{~mol}^{-1}\right)$. It is expected that at intermediate nitrogen pressures when no more interstitial nitrogen can be incorporated within the unit cell, $\mathrm{Fe}_{3} \mathrm{~N}$ or $\mathrm{Fe}_{2} \mathrm{~N}$ phases would be readily formed. In fact when sputtered with $\mathrm{R}_{N 2}=33 \%$, iron nitride structure is a mixture of $\mathrm{Fe}_{3} \mathrm{~N}$ and $\mathrm{Fe}_{2} \mathrm{~N}$ phases. Further increase in nitrogen partial pressure at $50 \%$, resulted in formation of nitrogen rich $\gamma^{\prime \prime \prime}$-type FeN. Still since the peaks were broadened, completely crystalline structure was not formed. At $\mathrm{R}_{N 2}=$ $83 \%$, sharp reflections corresponding to $\gamma^{\prime \prime \prime}$-FeN were observed. A further increase in the nitrogen partial pressure resulted in broadening of the XRD peaks. This means that well-defined polycrystalline structure of FeN compounds are only obtained at some specific nitrogen partial pressures and below and above these specific partial pressures the long range ordering is restricted due to incomplete Fe-N bonds or partial breaking of Fe- $\mathrm{N}$ bonds due to excessive nitrogen. Similar behavior was also observed for $\mathrm{NiFe}$, however amount of incorporation of nitrogen atoms in the two cases is different.

The energy of the adatoms with parameters used during sputtering for $\mathrm{Fe}$ or $\mathrm{NiFe}$ would be around $10 \mathrm{eV}, 56$ which corresponds to roughly $10^{5} \mathrm{~K}$. During condensation onto the substrate which tales place within $\sim$ msec, the adatoms are rapidly quenched, the mobility of the atoms is restricted; it is expected that either the occupancy of reactive nitrogen at interstitial sites or a chemical reaction between sputtered atom and nitrogen takes place in the plasma. Since the substrate were not heated intentionally, it is very unlikely that any rearrangement process would take place onto the substrate after condensation. This argument supports that microstructure of the deposited film would strongly depend on the plasma 
which in turn depend upon the amount of reactive nitrogen used during sputtering. Since nitrogen atoms gradually occupy interstitial sites in $\mathrm{Fe}$ or $\mathrm{NiFe}$, it is expected that the microstrain of the structure should increase as the amount of nitrogen partial pressure is increasing. The microstrain variation in the samples studied in the present case can be determined from XRD data using Williamson-Hall plots for Lorentzian peak shape: ${ }^{57}$

$$
b \cos \theta=A+B \sin \theta
$$

Where $A=0.9 \lambda / t, t$ is grain size and $B=4 \epsilon(\epsilon=$ microstrain), $b$ is the FWHM in $2 \theta$.

A straight line fit in the plot of $b \cos \theta$ and $\sin \theta$ yield the values of constant $A$ and $B$, which in turn yield the values of grain size as well as microstrain. Such a plot was obtained all the sample displayed in fig. 11 and fig. 4. The

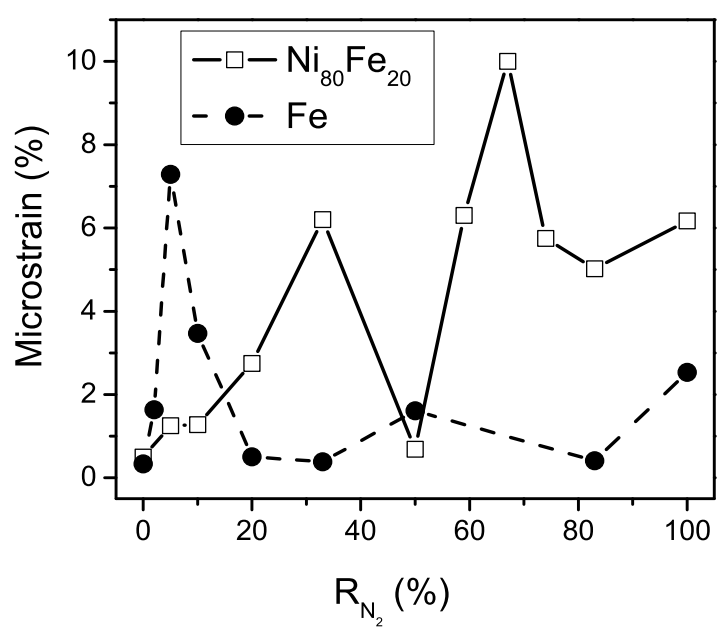

FIG. 15: Microstrain obtained from the XRD data (see fig. 1 and fig. (4) for FeN and NiFeN samples prepared with different nitrogen partial pressures. The solid or dotted line are guide to the eye.

obtained values of microstrain for $\mathrm{FeN}$ and $\mathrm{NiFeN}$ are plotted in fig. 15] It may be noted that the higher order reflections in the present case are reflected poorly, effective microstrain is obtained can be obtained using the most intense peak 58 Further, the microstrain in the deposited samples can have other origin such as grain size or dislocation. However a comparison of microstrain for the samples prepared at different nitrogen partial pressure is expected to provide additional insight on the mechanism inducing nanocrystallization or amorphization. It is interesting to see that for both FeN and NiFeN the microstrain is largest for the amorphous phases while for polycrystalline phases of nitrides or pure $\mathrm{Fe}$ and $\mathrm{NiFe}$, it is almost zero. An increase in the microstrain on nanocrystallization and amorphization gives an indication that the unit cell of $\mathrm{Fe}$ or $\mathrm{NiFe}$ is distorted due to incorporation of nitrogen at interstitial sites. Whenever a polycrystalline structure is formed, the microstrain is at minimum. Since the polycrystalline structure is formed due to almost complete covalent bonds between $\mathrm{Fe}$ and $\mathrm{N}$ or $\mathrm{NiFe}$ and $\mathrm{N}$, the distortion of the unit cell should be at minimum. An incomplete bonding or excessive nitrogen increased the microstrain in the film which leads to nanocrystalline or amorphous phase of the deposited sample. This argument combined with the fact that adatoms are quenched at the substrate within a very short time, explains the mechanism inducing nanocrystallization or amorphization. Even though amorphization or nanocrystallization increased the microstrain within the structure of the deposited film, it is interesting to note that surface roughness of the amorphous film was improved on amorphization, which gives an indication that strains are developed within the structure.

\section{CONCLUSIONS}

From the present study it can be concluded that reactive nitrogen sputtering of bcc-Fe and fcc-NiFe (at room temperature) forms a nanocrystalline or amorphous structure at most of the nitrogen partial pressures. Only at specific nitrogen partial pressures polycrystalline nitrides were obtained which are thermodynamically favored. Above or below this specific pressure the long range ordering is perturbed by reactive nitrogen. The crystallization in both amorphous FeN and NiFeN takes place in a single step and at higher temperature nitrogen poor phases are formed due to nitrogen out-diffusion. The growth behavior of amorphous FeN showed improved surface roughness due to amorphization and the growth exponent $\beta$ was minimum for amorphous phase as compared with poly or nanocrystalline phases. The magnetic measurements on ferromagnetic FeN and NiFeN films reveal that in case of $\mathrm{FeN}$, at low nitrogen content, the alloy forms a soft-magnetic phase while at higher nitrogen content the average value of magnetization decreased and coercivity increased. For NiFeN, inclusion of nitrogen produced phases with reduced values of magnetization. The magnetic moment of the samples was confirmed with polarized neutron reflectivity and was in agreement with the values obtained with DC extraction magnetometery.

\section{Acknowledgments}

We are grateful to Mr. Michael Horisberger for providing help with thin film deposition and Dr. Yu Lung Chiu for TEM measurements. One of author (RG), would like to thank Dr. Peter Allenspach for his support and availing the experimental facilities at Laboratory for Neutron Scattering, Paul Scherrer Institut. 
* Electronic address: mukul.gupta@psi.ch

1 C. A. Angell, K. L. Ngai, G. B. McKenna, P. F. McMillan, and S. W. Martin, J. Appl. Phys. 88, 3113 (2000).

2 P. G. Debenedetti and F. H. Stillinger, Nature 410, 259 (2001).

3 M. E. McHenry, M. A. Willard, and D. E. Laughlin, Prog. Mat. Sci. 44, 291 (1999).

4 A. Dunlop, G. Jaskierowicz, G. Rizza, and M. Kopcewicz, Phys. Rev. Lett. 90, 015503 (2003).

${ }^{5}$ C. Line, D. Sun, S. L. Ming, E. Jiang, and Y. Liu, Thin Solid film 279, 49 (1996).

${ }^{6}$ N. Sulitanu and F. Brinza, J. Opto Elec. Adv. Mat 5, 421 (2003).

7 F. Faupel, W. Frank, M. P. Macht, H. Mehrer, K. Rätzke, H. Schober, S. K. Sharma, and H. Teichler, Rev. Mod. Phys. 75, 237 (2003)

8 S. R. Elliott, Physics of Amophouse Materials (Longman Scientific and Tachnical, 1990), 2nd ed.

9 C. C. Koch, O. B. Cavin, C. G. McKamey, and J. O. Scarbrough, Appl. Phys. Lett. 43, 1017 (1983).

10 W. J. Meng, P. R. Okamoto, L. J. Thompson, B. J. Kestel, and L. E. Rehn, Appl. Phys. Lett. 53, 1820 (1988).

11 S. M. Sharma and A. K. Sikka, Prog. Mater. Sci. 40, 1 (1996).

12 R. B. Schwarz and W. L. Johnson, Phys. Rev. Lett. 51, 415 (1983).

13 W. L. Johnson, Prog. Mater. Sci. 28, 229 (1985).

14 K. Samwer, Phys. Rep. 161, 1 (1988).

15 M. Holz, P. Ziemann, and W. Buckel, Phys. Rev. Lett. 51, 1584 (1983).

16 S. Takeda and J. Yamasaki, Phys. Rev. Lett. 83, 320 (1999).

17 N. Hellgren, M. P. Johansson, E. Broitman, L. Hultman, and J.-E. Sundgren, Phys. Rev. 59, 5162 (1999).

18 D. Babonneau, M. Jaouen, M.-F. Denanot, P. Guérin, and F. Petroff, App. Phys. Lett. 82, 30565 (2003).

19 M. Gupta, A. Gupta, S. Rajagopalan, and A. K. Tyagi, Phys. Rev. B 65, 214204 (2002).

${ }^{20}$ A. Gupta, M. Gupta, B. A. Dasannacharya, S. Kikuta, Y. Yoda, and M. Seto, J. Jpn. Phys. Soc. 73, 423 (2004).

21 L. Rissanen, M. Neubauer, K. P. Lieb, and P. Schaaf, J. Alloys and Compds. 274, 74 (1998).

${ }^{22}$ M. A. Russak, C. V. Jahnes, E. Klokholm, J.-W. Lee, M. E. Re, and B. C. Webb, J. Appl. Phys. 70, 6427 (1991).

23 J.-F. Bobo, H. Chatbi, M. Vergnat, L. Hennet, O. Lenoble, P. Bauer, and M. Piecuch, J. Appl. Phys. 77, 5309 (1995).

${ }^{24}$ H. B. Nie, S. X. Xu, C. K. Ong, Q. Zhan, D. X. Li, and J. P. Wang, Thin Solid Films 440, 35 (2003).

25 T. Yoshitake and M. Ohkoshi, I.E.E.E. Trans. Magn 31, 3850 (1995)

26 M. Gupta, A. Gupta, P. Bhattacharya, P. Misra, and L. M. Kukreja, J. Alloys and Compds. 326, 265 (2001).

27 N. Wang, K. M. Ulmer, P. A. Constant, J. W. Anderegg, and J. E. Snyder, J. Vac. Sci. Technol. A 21, 1734 (2003).

28 L. Guibin, L. Guoqing, L. Minaki, and L. Bangzhi, Sur. Coat. Technol. 96, 34 (1997).

29 T. Kim and M. Takahashi, Appl. Phys. Lett. 20, 492 (1972).

30 Y. D. Zhang, J. I. Budnick, W. A. Hines, M. Q. Huang, and W. E. Wallace, Phys. Rev. B 54, 51 (1996).
31 C. Chang, J. M. Sivertsen, and J. H. Judy, I.E.E.E. Trans. Mag. MAG 23, 3636 (1987).

32 X. Wang, W. T. Zheng, H. Tian, S. S. Yu, and L. L. Wang, J. Magn. Magn. Mat. 283, 282 (2004).

33 M. Chiba, K. Morio, and Y. Koizumi, J. Magn. Magn. Mat. 242, 949 (2002).

34 M. Kawamura, Y. Abe, and K. Sasaki, Vacuum 59, 721 (2000).

35 M. Gupta, T. Gutberlet, J. Stahn, P. Keller, and D. Clemens, Pramana J. Phys 63, 57 (2004).

36 B. D. Cullity, Elements of X-ray Diffraction (AddisonWesley,MA, 1978).

37 A. Guinier, X-Ray Diffraction : In Crystals, Imperfect Crystals and Amorphous Bodies (Dover, New York, 1994).

38 K. Hono and D. H. Ping, Materials Characterization 44, 203 (2000).

39 D. M. Zhu, K. Raviprasad, K. Suzuki, and S. P. Ringer, J. Phys. D:Appl. Phys. 37, 645 (2004).

40 C. Braun, Parratt32- The Reflectivity Tool (HMI Berlin, 1997-99).

41 L. G. Parratt, Phys. Rev. 95, 359 (1954).

42 A. L. Barabási and H. E. Stanley, Fractal Concepts in Surface Growth (Cambridge University Press, 1995).

43 M. Lutt, J. P. Schlomka, M. Tolan, J. Stettner, O. H. Seeck, and W. Press, Phys. Rev. B 56, 4085 (1997).

44 M. Kardar, G. Parisi, and Y.-C. Zhang, Phys. Rev. Lett. 56, 889 (1986).

45 G. Herzer, I.E.E.E. Trans. Mag. 25, 3327 (1989).

46 G. Herzer, I.E.E.E. Trans. Mag. 26, 1397 (1990).

47 J. F. Loffler, J. P. Meier, B. Doudin, J.-P. Ansermet, and W. Wagner, Phys. Rev. B 57, 2915 (1998).

${ }^{48}$ R. W. Gao, W. C. Feng, H. Q. Liu, B. Wang, W. Chen, G. B. Han, P. Zhang, H. Li, W. Li, Y. Q. Guo, et al., J. Appl. Phys. 94, 664 (2003).

49 J. F. Loffler, H.-B. Braun, and W. Wagner, Phys. Rev. Lett. 85, 1990 (2000).

50 H. Hoffmann and T. Fujii, J. Magn. Magn. Mat 128, 395 (1993).

51 B. D. Cullity, Introduction to Magnetic Materials (Addison-Wesley,MA, 1972).

52 S. J. Blundell and J. A. C. Bland, Phys. Rev. B 46, 3391 (1992).

53 S. Hope, J. Lee, P. Rosenbusch, G. Lauhoff, J. A. C. Bland, A. Ercole, D. Bucknall, J. Penfold, H. J. Lauter, V. Lauter, et al., Phys. Rev. B 55, 11422 (1997).

${ }^{54}$ F. Ott, SIMULREFLEC, http://www-llb.cea.fr/prism/programs/simulreflec/ simulreflec.html.

${ }^{55}$ F. Tessier, A. Navrotsky, R. Niewa, A. Leineweber, H. Jacobs, S. Kikkawa, M. Takahashi, F. Kanamaru, and F. J. DiSalvo, Solid State Sciences 2 (2000).

${ }^{56}$ G. M. Turner, I. S. Falconer, B. W. James, and D. R. McKenzie, J. Vac. Sci. Technol. A 10, 455 (1992).

57 Y. Rosenberg, V. S. Machavariani, A. Voronel, S. Garber, A. Rubshtein, A. I. Frenkel, and E. A. Stern, J. Phys.: Condens. Matter 12, 8081 (2000).

${ }^{58}$ L. R. Shaginyan, M. Misina, J. Zemek, J. Musil, F. Regent, and V. F. Britun, Thin Solid Films 408, 136 (2002). 